Case Report

\title{
Non-surgical Furcal Perforation Management with Biodentine in Retreatment: A 1-Year Recall Review
}

CT Valliappan ${ }^{1}$, J Joyson Joe Asir ${ }^{1}$, V Subashri ${ }^{1}$, V Vaishnavi ${ }^{2}$, I Anand Sherwood ${ }^{3,}$ *

1. Post graduate students, Department of Conservative Dentistry and Endodontics, CSI College of Dental Sciences, Madurai, Tamil Nadu, India; E-Mails:vrvalliappan2@gmail.com; joysonjoe10@gmail.com; shrimitha26@gmail.com

2. Private practitioner, Chennai, Tamil Nadu, India; E-Mail:vaishnavivenkat@yahoo.com

3. Professor and Head, Department of Conservative Dentistry and Endodontics, CSI College of Dental Sciences, Madurai, Tamil Nadu, India; E-Mail: anand.sherwood@gmail.com

* Correspondence: I Anand Sherwood; E-Mail: anand.sherwood@gmail.com

Academic Editor: Hossein Hosseinkhani

Special Issue: Research on Dental Materials

Recent Progress in Materials

2021, volume 3, issue 2

doi:10.21926/rpm.2102014
Received: December 28,2020

Accepted: April 21,2021

Published: April 27,2021

\begin{abstract}
Furcal perforation management in root canal retreatment is often challenging as the outcome of the perforation sealing depends on several parameters. The recent introduction of several bioactive cements and magnification systems has made the sealing of perforations easier and the outcome more predictable. This study highlights the clinical outcomes of furcal perforation management in root canal retreatment using biodentine.
\end{abstract}

\section{Keywords}

Bioactive cements; biodentine; operating microscope; perforation; root canal retreatment

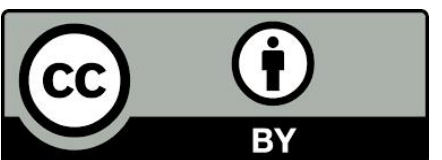

(C) 2021 by the author. This is an open access article distributed under the conditions of the Creative Commons by Attribution License, which permits unrestricted use, distribution, and reproduction in any medium or format, provided the original work is correctly cited. 


\section{Introduction}

Furcal perforations represent pathologic or iatrogenic communication between the root canal space and the attachment apparatus [1].Variables considered duringthe management of furcation include level,location, size, and time of perforation[1].In root canal retreatment, the presence of perforation would not always be visible on the pre-treatment radiograph but confirmed during retreatment only [2-4]. A dental operating microscope has become a standard instrument to predictably and non-surgically repair perforation defects [1]. Materials recommended for sealing of perforation defects include, but are not limited to, hemostatics, resorbable and non-resorbable barriers [1]. Bio-active glass materials have shown excellent biocompatibility in perforation repair [5]. Biodentine is a calciumsilicate-based cement created as dentin support with additional advantages over mineral trioxide aggregate (MTA). Its advantages arebetter handling characteristics, self-adhesion to dentin, no shrinkage, better anti-bacterial activity, and induction of hard tissue formation [1]. The use of biodentineis not limited to furcal perforation, and it has successfully been applied in other clinical situations during endodontic treatment [6].

The following case reports discuss the application of biodentine in non-surgical furcal perforation management during root canal retreatment and highlight the predictable clinical successes achieved. All patients were treated after obtaining written informed consent.

\section{Case 1}

A 25-year-old male patient presented for post-endodontic restoration in a previously root canal treated mandibular left first molar (Figure 1A). Clinical examination showed significant loss of tooth structure; therefore, a fiber post composite core followed by a full-coverage crown was planned. During post preparation in the distal canal, a strip perforation occurred in the middle third of the canal close to the furcation. The perforation was managed by placing biodentine (Septodont Healthcare India Pvt Ltd., Raigad, India) guided by an operating microscope (Labomed Prima, Labomed Inc., CA, USA). This was followed by the application of endocrown (IPS Emax, IvoclarVivadent AG, Liechtenstein) as a post-endodontic restoration (Figure 1B-H). An immediate post-perforation radiograph showed furcal bone loss (Figure 11). One month after perforation management, the patientpresented with a periodontal abscess with respect to the treated teeth with further deterioration of the furcal defect in the radiograph (Figure 2A, B). The gingival overgrowth and periodontal abscess tissue were excised using a laser (iLase, Biolase, CA, USA). This was followed with gingival sulcus curettage using an ultrasonic scaler (Figure 2C). A clinical review at 14-month recall revealed an excellent clinical periodontal healing with no attachment loss, and radiographic examination showed stabilization of the bone loss at the perforation site (Figure 2D, E). 


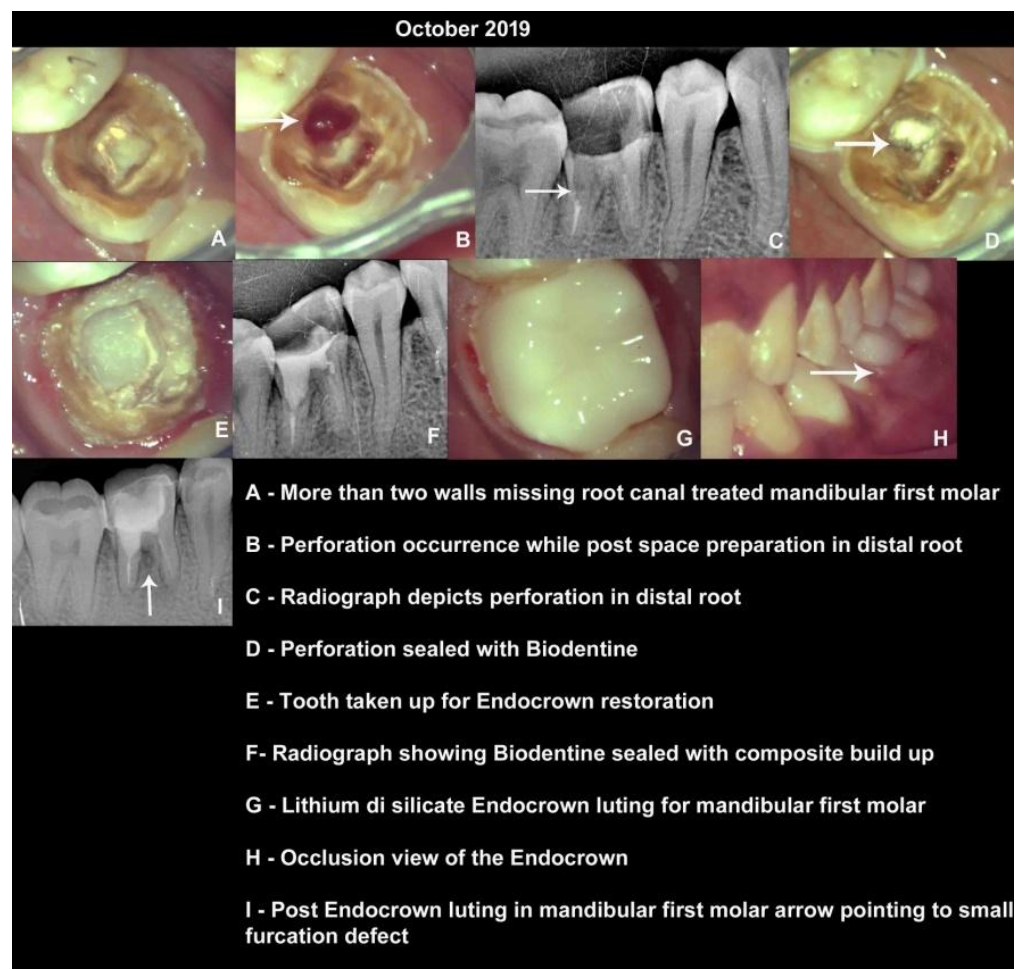

Figure $1 \mathrm{~A}$. Pre-restoration image of mandibular first molar showing extensive crown structure loss. B. Strip perforation (arrow) in the distal canal during post space preparation. C. Radiograph depicting the perforation (arrow). D. Perforation sealed with biodentine (arrow). E. Tooth prepared for endocrown restoration. F. Radiograph showing strip perforation sealed in the distal canal. G. Immediate post endocrown restoration image. H. Lithium disilicate endocrown restoration (arrow) in occlusion. I. Immediate post endocrown restoration radiograph with perforation sealed (arrow).

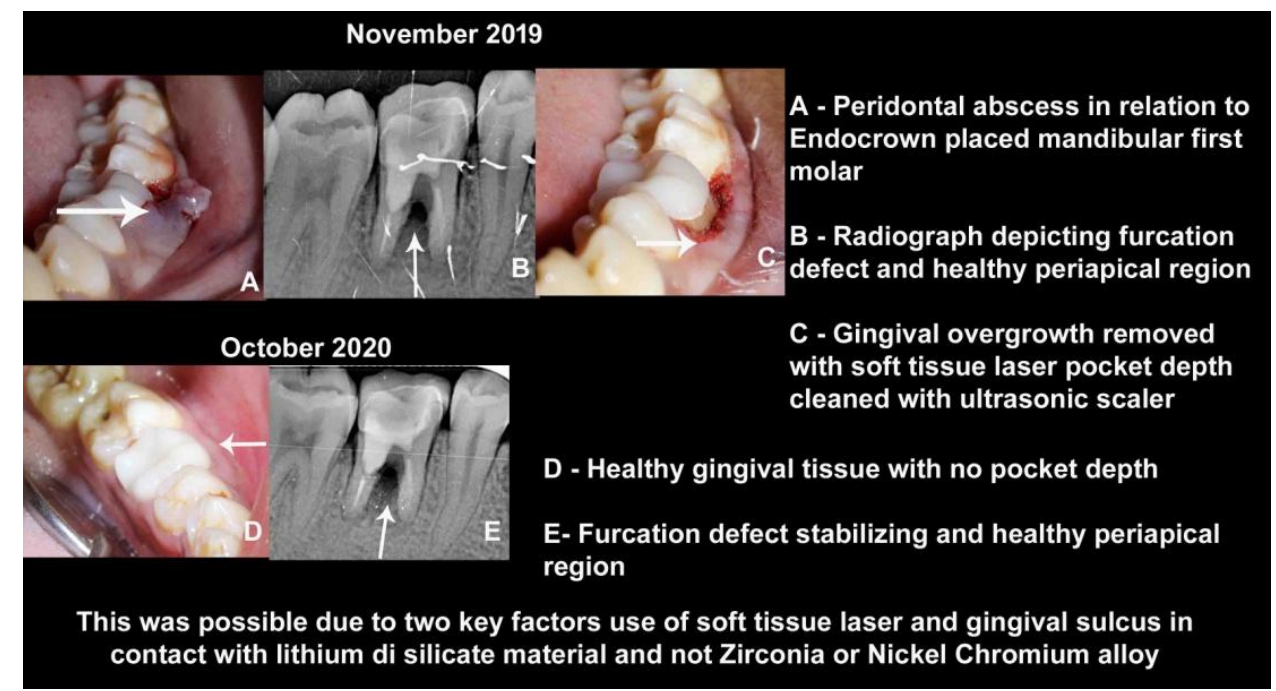

Figure 2 A. Periodontal abscess in lithium disilicate endocrown restored mandibular first molar 1 month after perforation sealing. B. Furcation bone loss (arrow) in endocrown placed mandibular first molar. C. Laser excision of abscess tissue (arrow). D. Normal gingival attachment 1 year after laser-tissue excision(arrow). E. Furcation bone stabilized (arrow) after 1 year. 


\section{Case 2}

A 40-year-old male patient presented to the Department of Endodontics with a chief complaint of pain in his previously root canal treated right mandibular first molar. Radiographic examination showed inadequate obturation of both mesial and distal canals; therefore,root canal retreatment was suggested (Figure 3A). Upon removal of gutta-percha (GP) and refining the access cavity, a perforation defect was noted at the floor of the pulp chamberthat was not obvious in the pretreatment radiograph (Figure 3B, C). After complete removal of the existing gutta-percha from the canals, the canals were re-obturatedafter1week of disinfection with intracanal medicament (Figure 3D, E). The perforation defect was closed with biodentine under an operating microscope (Figure 3F). Figure 3G showsan immediate assessment of perforation defect in the radiograph,whereas Figure $3 \mathrm{H}$ shows an excellent furcal bone healing in the biodentine-treated site in a 17-months radiograph.

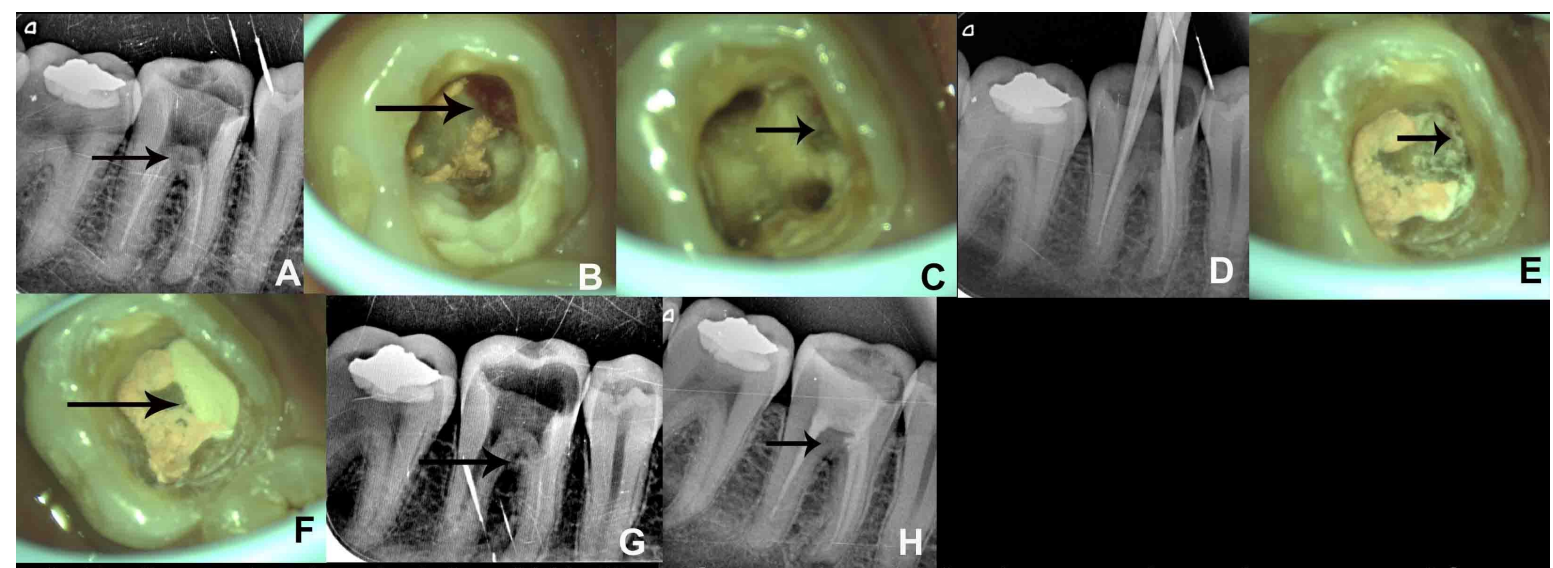

A- Radiograph taken in 8 Apr 2019. Mand first molar with inadequete obturation reported for retreatment. With very little evidence present in radiograph about perforation.

B - Upon gutta percha removal only perforation was evident. This is evidence from studies by Sherwood 2012, Hoen and Pink 2002 that a single pre operative radiograph might not be able to detect all the defects present in retreatment .

C- Gutta perach removed and shaping of canals accomplished rotary nickel titanium files. D \& E - Gutta percha obturation done.

F - Perforation sealed with Biodentine covered with glass ionomer and coronal restoration with composite in same appointment

G - Radiograph of tooth 17 Apr 2019 on day of obturation and perforation sealing.

H - Radiograph taken on 22 Oct 2020 post lockdown arrow depict excellent healing of the furcation perforation patient has no complaint. Also you can observe that tooth structure is intact even without crown placement

Figure $3 \mathrm{~A}$. Pre-treatment radiograph of failing root canal treatment with inadequate obturation and no obvious perforation evidence (arrow). B and C. Following access refining and gutta-percha removal from the canals,clear perforations (arrow) were observed on the lingual side of the pulp floor. D. Following gutta-percha removal, master cone selection was performed. E. Canals obturated with gutta-percha. F. Perforation site sealed with biodentine (arrow). G. Radiograph showing gutta-percha removed from the canals. $\mathrm{H}$. An 18-month post perforation management radiograph showing no furcal bone loss. 


\section{Case 3}

A 37-year-old male patient presented with complaints of pain in his failing root canal treated left mandibular first molar. Radiographic examination showed obturation beyond apex and instrument separation in the mesial canal (Figure 4A). In addition, periapical lesions were evident in the mesial and distal canals (Figure 4A). Root canal retreatment and retrieval of the separated instrument were planned. After removal of gutta-percha from the mesiolingual and distal canals, the canals were re-obturated after 1-week disinfection with intracanal medicament (Figure 4B-D). Instrument retrieval in the mesiobuccal canal was performed with an instrument removal system(IRS, Swiss Machining, San Diego, CA, USA) under magnification. The core drills, used to create a platform in the system to remove the separated instrument, created a strip perforation in the middle third of the canal close to the furcation (Figure 4E, F). Following perforation, the instrument retrieval attempt was abandoned and the canal space apical to perforation defect was obturated with thermoplasticized gutta-percha (Super Endo Alpha, B and L Biotech Inc, US) (Figure 4G). Perforation defect and canal space coronal to perforation were sealed with biodentine (Figure 4H, I). A 12-month review showed excellent gingival health with no pocket depth.Radiograph examination showed good periapical healing (Figure 4J-K).

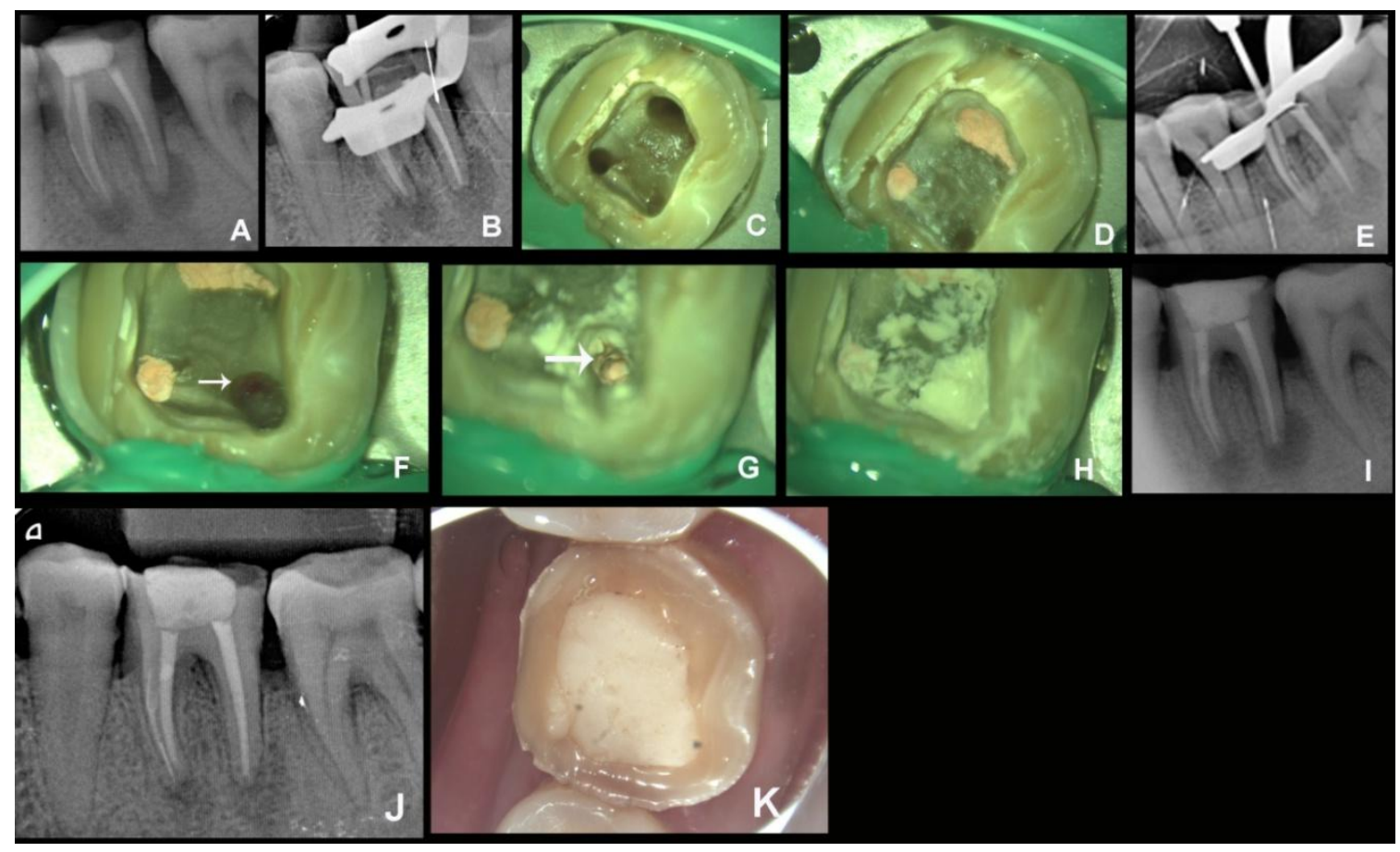

Figure 4 A. Pre-operative radiograph showing failed root canal treated mandibular first molar with the separated instrument in the mesial canal and periapical radiolucency in both mesial and distal root canals. B. Radiograph showing an attempt to remove guttapercha. C. Gutta-percha was removed from the root canals. D.Mesiolingual and distal canals obturated before attempting separated instrument retrieval from the mesiobuccal canal. E. IRS drill used for staging and creating the platform.F. Strip perforation (arrow) in the mesiobuccal canal because of IRS drill usage. G. Canal space apical to perforation site filled with thermoplasticized gutta-percha. H. Perforation site and canal space coronal to perforation sealed with biodentine (arrow). I. Post 
perforation management radiograph. J.One-year post-treatment radiograph showing no furcal bone, and periapical lesion shows healing. K.One-year post perforation management image of the mandibular first molar showing no gingival attachment loss.

\section{Case 4}

A 40-year-old female patient presented to the endodontic clinic with complaints of pain in her recently root canal treated left mandibular first and second molar. Radiographic examinationrevealed multiple defects in the treated root canals in both molars (Figure 5A). It was evident in the radiograph that first and second molars had over-extended access cavities, instrument separation beyond apex, gutta-percha placement in furcal perforation, and had missed canals (Figure 5A). Multiple defects were explained to the patient and an option of non-surgical retreatment was suggested. Retreatment and gutta-percha removal were performed in the mandibular second molar, followed by the first molar. Gutta-percha points were removed from both the molars (Figure 5B), but the separated hand instrument located beyond the apex in the mesial canals of the mandibular first molar could not be retrieved (Figure 5B, C). Multiple pulpal floor perforations in the furcal region with over-extended access cavities were observed under an operating microscope in both molars (Figure 5D, E, H, K, L). Following obturation of the canal system with gutta-percha after disinfection with intra-canal medicament, the pulpal floor perforations were sealed with biodentine. Figure $5 \mathrm{~N}$ shows an immediate post-obturation radiograph of the two molars.

Six months after retreatment,the patient reported back with a fractured lingual crown of the mandibular first molar tooth that had occurred 3 months after perforation repair (Figure 50, P). This resulted from the over-extended access cavity from the initialroot canal treatment. Gingival tissue overgrowth into the fractured portion of the molar was excised using laser, followed by fiber post composite core build-up (Reforpost, Angelus Industrial Products, Londrina, Brazil) (Figure 5Q, R, T). Following post-placement, the re-exposed perforation sites were sealed with biodentine and composite (Charisma Smart Composite, Kulzer GmbH, Germany) core build-up was performed (Figure $5 \mathrm{U}-\mathrm{W}$ ). The patient was recalled 9 months after fracture of her mandibular first molar tooth, the tooth was asymptomatic, and periodontal probing exhibited pocket presence on the lingual aspect of teeth with bleeding upon probing (Figure $5 \mathrm{X}, \mathrm{Y}$ ). Radiograph examination showed no furcal bone loss in the second molar; however, the first molar exhibited a certain amount of furcal bone loss due to an earlier fracture of the crown portion (Figure 5Z). A 15-month review of teeth showed the clinical success of the mandibular second molar with good periodontal health. A review at9-month recall after crown fracture management revealed the surviving tooth. However, clinical success can be re-assessed over a longer period. 


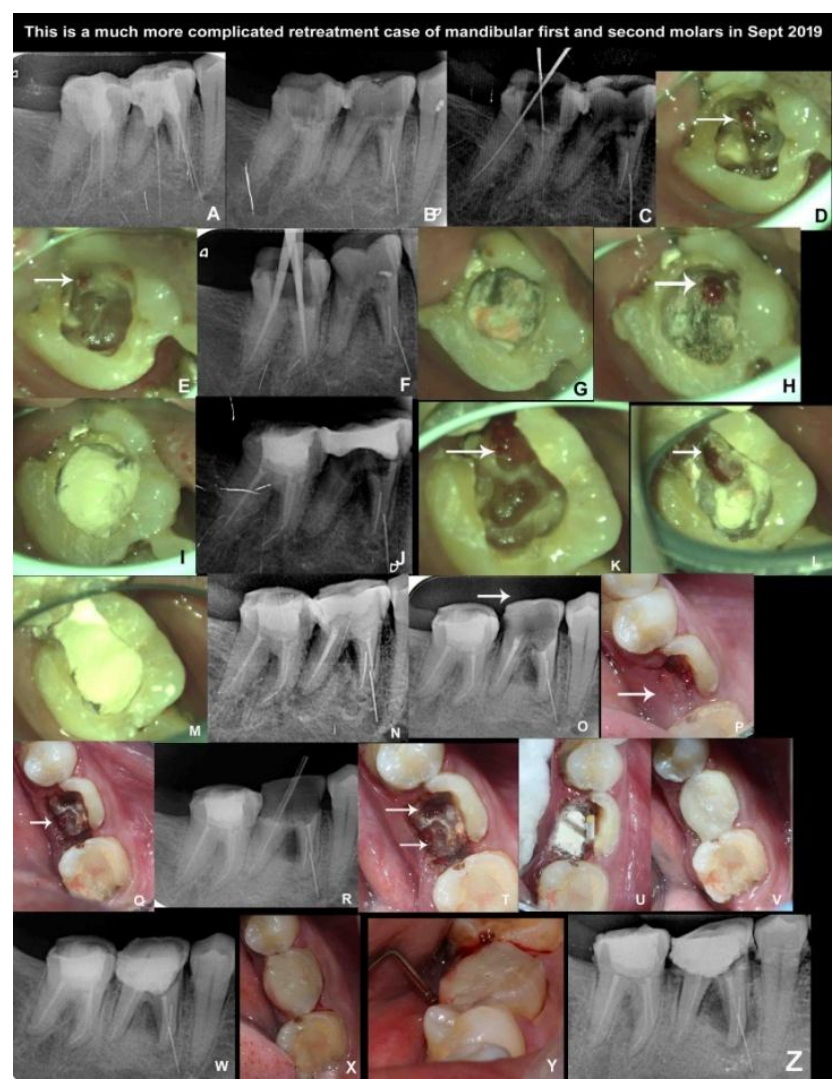

Figure $5 \mathrm{~A}$. Pre-treatment radiograph depicting multiple defects such as missed canals and instrument separation beyond apex in left mandibular first and second molars. B. Radiograph showing complete gutta-percha removal from the canals with separated instrument present in the mesial canal of the mandibular first molar. C. Working length estimation radiograph. $D$ and $E$. Multiple perforations (arrow) detected in mandibular first and second molars. F. Master selection radiograph. G. Canals obturated in mandibular second molar. H. Perforation (arrow) site ready for biodentine sealing after canal obturation. I. Perforation site sealed with Biodentine. J. Post perforation sealed radiograph of mandibular second molar. K. Perforation (arrow) in mandibular first molar. L. Canals obturated before perforation (arrow) sealing. M. Perforation sealed with Biodentine. N. Post perforation sealed mandibular first and second molar radiograph. $\mathrm{O}$. Six months post perforation management radiograph displaying fracture crown structure in mandibular first molar (arrow). P. Gingival tissue overgrowth (arrow) covering the pulpal floor. Q. Laser tissue excision of the gingival tissue (arrow) showing extensiveness of the crown structure loss. R. Fiber post placement in distal canal radiograph. T. Earlier perforation (arrow) sealed sites exposed because of the crown structure fracture. $U$. The perforation resealed with biodentine following fiber post placement in distal canal. V. Composite core buildup of mandibular first molar. W. Immediate resealed perforation radiograph. X. Mandibular first molar 9 months after resealing of perforation. Y. Lingual aspect of mandibular first molar exhibiting periodontal pocket formation. Z. The 15-month post perforation management of mandibular second molar showing no furcal bone loss, 9-month post perforation resealed mandibular first molar some furcal bone loss. 


\section{Case 5}

A 53-year-old female patient reported complaints of pain in her root canal treated left maxillary second premolar (Figure 6A). Radiographic examination showed an ill-fitting full-coverage crown and inadequately root canal treated maxillary second premolar, with the widening of the periodontal ligament space (Figure 6A). Upon removal of the crown and gutta-percha, a coronal perforation was identified on the mesial aspect of the access cavity, which was not evident in the pre-treatment radiograph (Figure 6B). Following treatment with intracanal medicament, the canal was thermoplastically obturated with gutta-percha, and the perforation site was sealed with biodentine (Figure 6C-E). The access cavity was restored with amalgam.

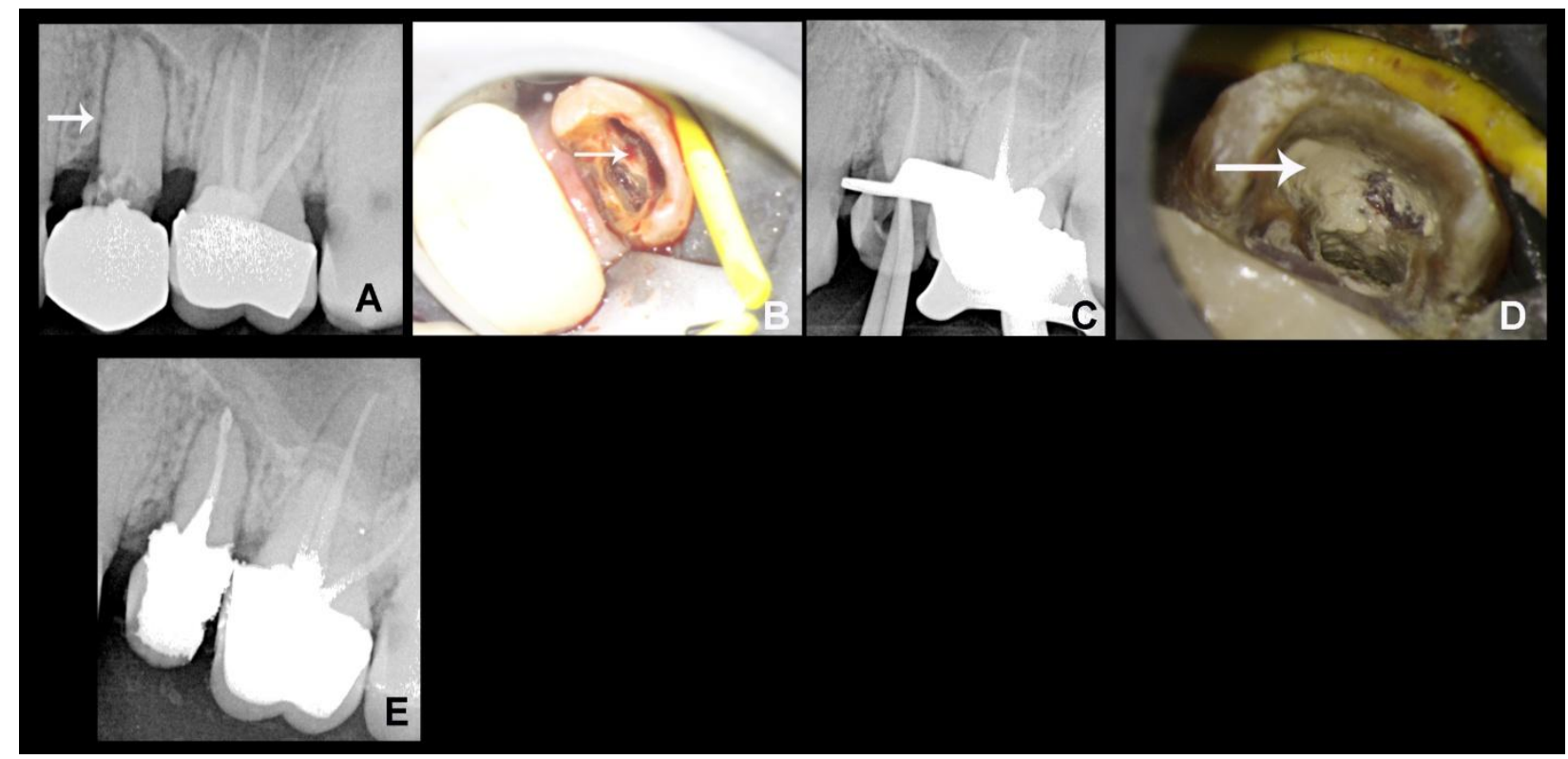

Figure $6 \mathrm{~A}$. Pre-treatment radiograph of failing root canal treated left maxillary second premolar with the full-coverage crown. B. Following crown removal and the guttapercha removal from the canals, a perforation (arrow) was detected in the mesial aspect of the pulpal floor. C. Master cone selection radiograph. D. Perforation site sealed with biodentinefollowing obturation of canals. E. Radiograph of immediate post perforation management with amalgam core build up in the maxillary second premolar.

\section{Case 6}

A 52-year-old female patient presented to the clinic with pain in her attempted root canal treated left maxillary first molar. Radiographic examination showed a previously prepared access cavity with possible perforation in the pulpal floor close to furcation (Figure 7A). Root canals were cleaned, shaped, and subsequently obturated after intracanal medicament. Subsequently, the perforation was sealed with biodentine and access preparation was restored with composite (Figure 7B-F). 


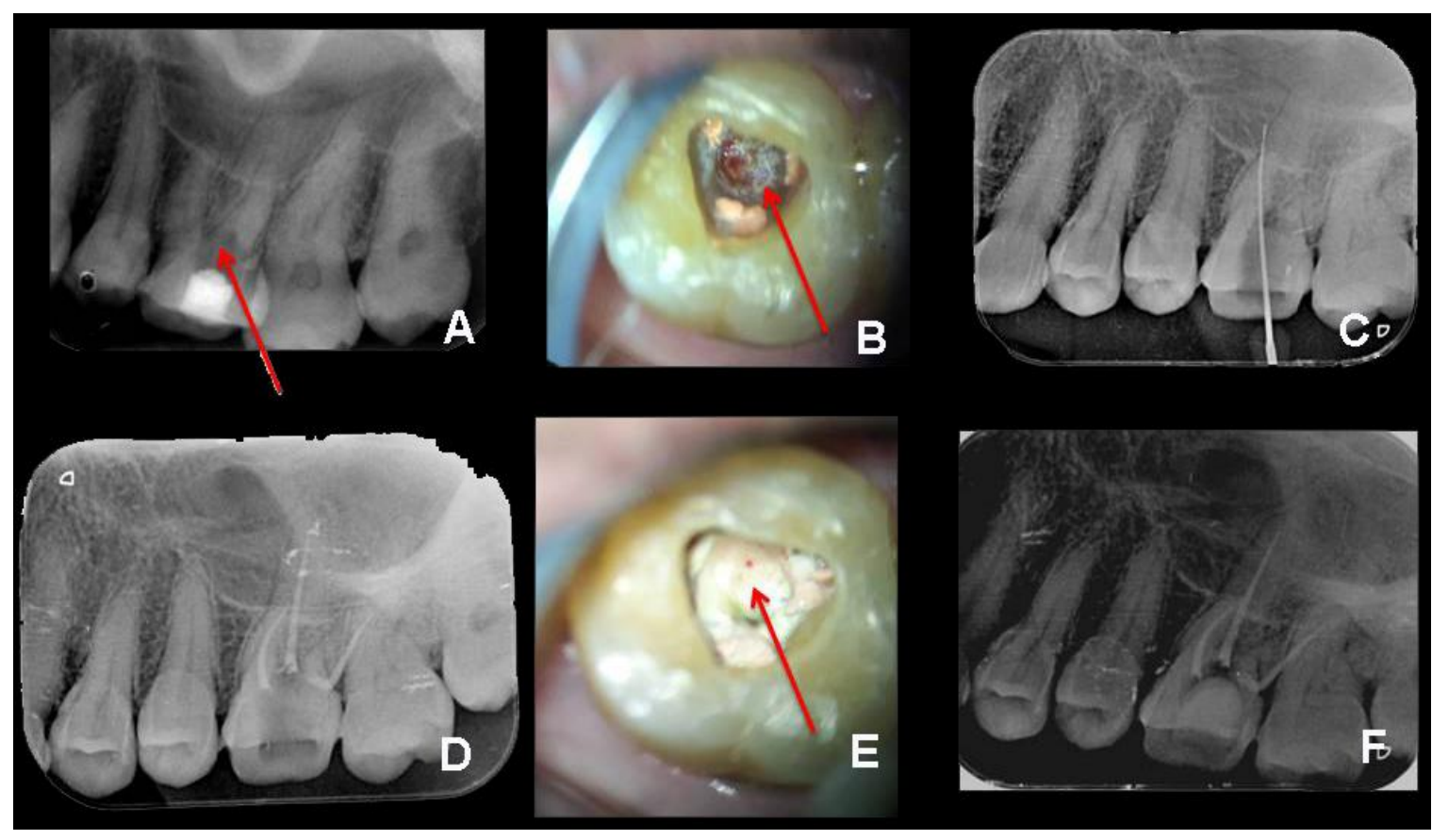

Figure 7 A. Pre-treatment radiograph of a left maxillary first molar with history of root canal treatment displaying possible perforation (arrow). B. Radiograph displaying the presence of perforation in the furcal region. C.Obturation of management canals before perforation (arrow). D. Radiograph of canals obturated before perforation sealing. E. Perforation site sealed with biodentine (arrow). F. Immediate post perforation management radiograph.

\section{Case 7}

A 32-year-old female patient presented with a painful root canal treated left maxillary first molar, with a dislodged full-coverage crown. Radiographic examination showed inadequate root canal treatment (Figure 8A); therefore, non-surgical root canal retreatment was planned. Upon removal of gutta-percha from the root canals, a pulpal floor perforation was observed in the furcation region which was not obvious in the pre-treatment radiograph (Figure 8B). Perforation sealing with biodentine was performed after root canal obturation with gutta-percha (Figures 8CE). Following root canal treatment, the tooth had a laser surgical crown lengthening on buccal and palatal regions andwas restored with a full-coverage zirconia crown (Figure $8 \mathrm{~F}, \mathrm{G}$ ). Reviews with radiographs at 5 and 18 months revealed no evidence of furcal bone loss (Figure $8 \mathrm{H}, \mathrm{I}$ ). Figures $8 \mathrm{~J}$ and $\mathrm{K}$ are clinical photographs of the molar tooth restored with zirconia crown with excellent gingival and periodontal health. 


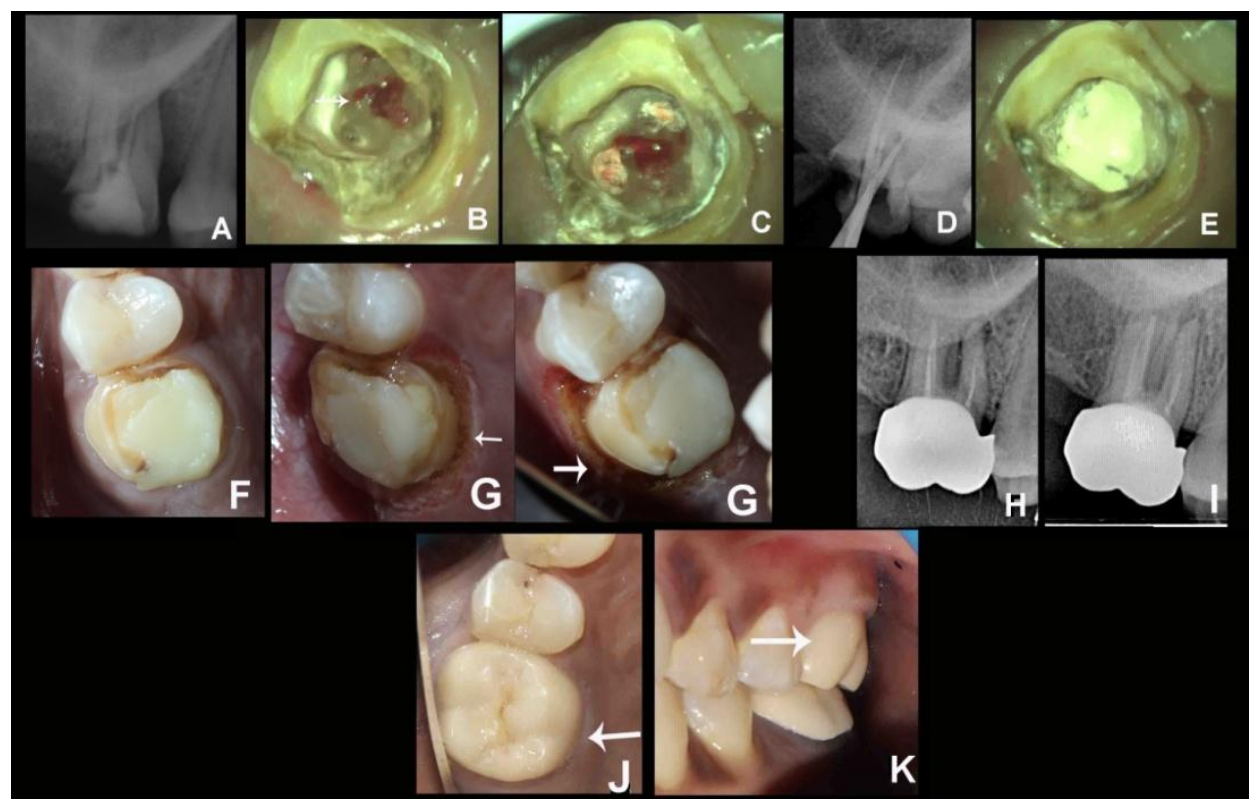

Figure $8 \mathrm{~A}$. Pre-treatment radiograph of the left maxillary first molar tooth. B. Evidence of perforation (arrow) observed in the pulpal floor in the furcal region. C. Following removal of previous gutta-percha fillings, the root canals were re-obturated with gutta-percha points. D. Radiograph showing placement of new gutta-percha points. $E$. Perforation site sealed with biodentine following obturation of canals. F. Post endodontic composite restoration to seal the access preparation with inadequate crown length. G. Laser crown lengthening performed on palatal (arrow) and buccal (arrow) aspect of maxillary first molar. $\mathrm{H}$ and I. Five-and 18-month review radiographs, respectively, showing no furcal bone loss. J. An 18-month occlusal view of left maxillary first molar restored with a full-coverage zirconia crown exhibiting good gingival health in laser crown lengthening region (arrow). K. An 18-month buccal view of left maxillary first molar restored with a full-coverage zirconia crown exhibiting good gingival health in laser crown lengthening region (arrow).

\section{Discussion}

Perforation management in retreatment cases is largely complicated by the presence of other mishaps, such as instrument separation, inadequate shaping, and cleaning of either the canals, previous root canal fillings, or periapical lesions, as highlighted by the cases presented in this report. Several clinical studies have reported a remarkable success rate (90\%) achieved with MTA sealing of perforation defect [7-10].Pontius et al. [11] analyzed the clinical outcomes of 70 perforation managementcases in 69 patients and reported no significant difference in the success rate between primary and secondary root canal treatments and between the presence and absence of apical periodontitis [10]. The current case report finding is in agreement with the abovefindings of good clinical success by Pontius et al.Although biodentine, as a new bioceramic material, was introduced a decade ago in 2010, there are limited long-term clinical studies available in the literature on its performance in the management of retreatment cases [11].Case reports available in the literature point to a favorable outcome for biodentine similar to MTA [11]. However, biodentine has advantages over MTA in terms of better maneuverability and faster 
setting [11]. One drawback with biodentine is its lack of radiopacity, which might cause difficulty in detecting this material in radiographs. Several long-term studies $[9,12]$ on perforation management with MTA suggest successful healing; however, more clinical studies are required on the use of biodentine in perforation management.

One of the main critical factors in perforation management in root canal retreatment is the time and level of perforations[1], as the perforations might have occurred long back at the initial root canal treatment. Chronic perforations pose treatment challenges requiring surgical interventions [1].However, in the present report, all perforations except in Cases 1 and 3occurred at the time of primary root canal treatment and were managed with non-surgical interventions except for Cases 1 and 4. These cases (1 and 4) required surgical intervention for perforation repairs that are close to furcation. This is in concurrence with earlier suggestions that furcal perforations could threaten the sulcular attachment than apical perforations [1]. Case 1 had better periodontal healing with lithium disilicate ceramic endocrownfull-coverage crown when compared with Case 4 for two reasons; first, perforation closure was performed immediately at perforation detection whereas in Case 4 perforation was exposed to the oral environment and was left unattended for three months. Second, an inert ceramic material was in contact with sulcular attachment than composite. All other six cases except Case 2 had perforations close to the furcation. Of these, two teeth that exhibited extensive tooth structure loss required surgical laser gingival tissue excision. The present report highlights that furcal perforations in teeth with extensive tooth structure loss might require minimal surgical intervention. This is because of the long-term exposure of these sites to oral microbes that could prevent satisfactory healing of the periodontal tissues. All teeth in this report survived beyond 1year, even those having furcal perforations with extensive coronal structure loss. Pontius et al.[10] recommended thatteeth with chronic apical periodontitis and perforation healing could be reviewed in 1 year; however, a 2-year assessment would be better. Our experience in the management of perforation in root canal retreatment showed that a failed treatment tended to occur before 1 year. However, the affected teeth, which remained functional and asymptomatic even after 18months of retreatment,was found to be successful for a long period.

The present case reports show the versatility of biodentine, allowing it to be applied in complicated retreatment cases and achieve predictable clinical success, even in compromised perforation management. Recent magnification tools such as operating microscopes have allowed more precise management of perforation cases in root canal retreatment. The present report highlights the amount of coronal tooth structure loss in retreatment cases, with furcal perforations influencing the requirement for surgical intervention. Long-term,well-designed clinical trials are required for better assessment of biodentine as furcal perforation sealing material in root canal retreatment for teeth with and without extensive coronal tooth structure loss.

\section{Acknowledgments}

Authors would like to thank Dr Bennett. T. Amaechi BDS, MS, PhD, MFDS RCPS (Glasg), FADI, Professor and Director of Cariology, Department of Comprehensive Dentistry University of Texas Health, San Antonio, USA for his help with language editing of the manuscript. 


\section{Author Contributions}

Valliappan CT, Joyson Joe Asir J, Subashri V, Vaishnavi V - Manuscript preparation, Patient management. I Anand Sherwood - Manuscript preparation, editing, conceptualization of the idea, follow-up of patients.

\section{Competing Interests}

The authors have declared that no competing interests exist.

\section{References}

1. Machtou P, Ruddle CJ. Endodontic orthograde retreatment and management of mishaps. In: Ingle's endodontics. 7th ed. Raleigh, NC: PMPH US; 2019.pp.729-796.

2. Sherwood IA. Pre-operative diagnostic radiograph interpretation by general dental practitioners for root canal treatment. DentomaxillofacRadiol. 2012; 41: 43-54.

3. Hoen MM, Pink FE. Contemporary endodontic retreatments: An analysis based on clinical treatment findings. J Endod. 2002; 28: 834-836.

4. Valliappan $C T$, Sherwood IA, Farooq M, Aarthi V. Procedural errors observed with primary root canal treatment delivery in teeth taken for non-surgical retreatment in a dental college from Southern India.ContempClin Dent. 2021.

5. Samiee M, Eghbal MJ, Parirokh M, Abbas FM, Asgary S. Repair of furcal perforation using a new endodontic cement. Clin Oral Investig. 2010; 14: 653-658.

6. Sherwood Al, Gutmann JL, Deepika G, Sadashivam V, J E, Meena D, et al. Biodentine: A unique bio-active endodontic material with versatile uses. J Dent Health Oral Res. 2020; 1: 1-15.

7. Mente J, Hage N, Pfefferle T, Koch MJ, Geletneky B, Dreyhaupt J et al. Treatment outcome of mineral trioxide aggregate: Repair of root perforations. J Endod. 2010; 36: 208-213.

8. Mente J, Leo M, Panagidis D, Saure D, Pfefferle T. Treatment outcome of mineral trioxide aggregate: Repair of root perforations-long-term results. J Endod. 2014; 40: 790-796.

9. Siew K, Lee AH, Cheung GS. Treatment outcome of repaired root perforation: A systematic review and meta-analysis. J Endod. 2015; 41: 1795-1804.

10. Pontius V, Pontius O, Braun A, Frankenberger R, Roggendorf MJ. Retrospective evaluation of perforation repairs in 6 private practices. J Endod. 2013; 39: 1346-1358.

11. Kaur M, Singh H, Dhillon JS, Batra M, Saini M. MTA versus Biodentine: Review of literature with a comparative analysis. J Clin Diagn Res. 2017; 11: ZG01-ZG05.

12.Gorni FG, Andreano A, Ambrogi F, Brambilla E, Gagliani M. Patient and clinical characteristics associated with primary healing of iatrogenic perforations after root canal treatment: Results of a long-term Italian study. J Endod. 2016; 42: 211-215. 


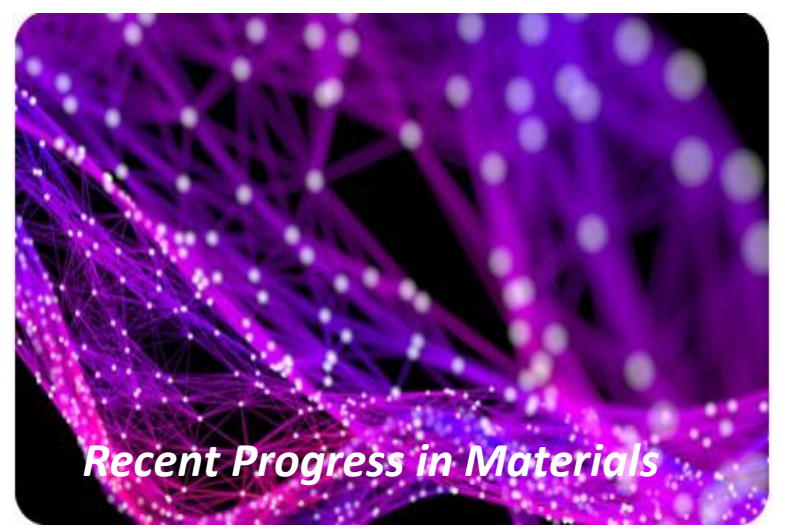

Enjoy Recent Progress in Materials by:

1. Submitting a manuscript

2. Joining in volunteer reviewer bank

3. Joining Editorial Board

4. Guest editing a special issue

For more details, please visit: http://www.lidsen.com/journals/rpm 